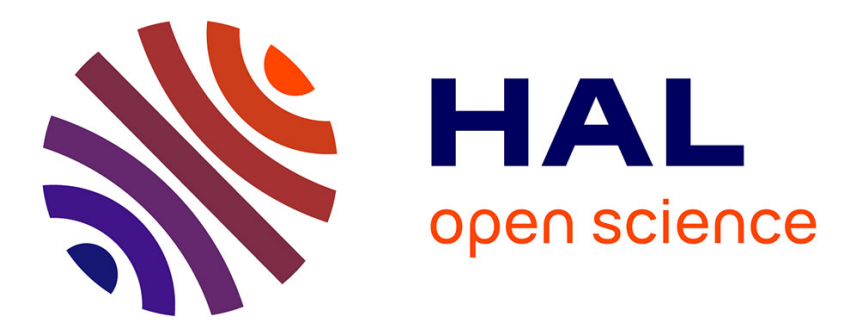

\title{
Recovery of 3-hydroxypropionic acid from organic phases after reactive extraction with amines in an alcohol-type solvent
}

\author{
F. Chemarin, M. Moussa, F. Allais, Ioan-Cristian Trelea, V. Athes
}

\section{- To cite this version:}

F. Chemarin, M. Moussa, F. Allais, Ioan-Cristian Trelea, V. Athes. Recovery of 3-hydroxypropionic acid from organic phases after reactive extraction with amines in an alcohol-type solvent. Separation and Purification Technology, 2019, 219, pp.260-267. 10.1016/j.seppur.2019.02.026 . hal-02560888

\section{HAL Id: hal-02560888}

\section{https://hal-agroparistech.archives-ouvertes.fr/hal-02560888}

Submitted on 2 May 2020

HAL is a multi-disciplinary open access archive for the deposit and dissemination of scientific research documents, whether they are published or not. The documents may come from teaching and research institutions in France or abroad, or from public or private research centers.
L'archive ouverte pluridisciplinaire $\mathbf{H A L}$, est destinée au dépôt et à la diffusion de documents scientifiques de niveau recherche, publiés ou non, émanant des établissements d'enseignement et de recherche français ou étrangers, des laboratoires publics ou privés. 


\title{
Recovery of 3-hydroxypropionic acid from organic phases after reactive extraction with amines in an alcohol-type solvent
}

\author{
F. Chemarin ${ }^{a, b}$, M. Moussa ${ }^{b^{*}}$, F. Allais ${ }^{a, b}$, I.C. Trelea ${ }^{b}$, V. Athès ${ }^{b}$

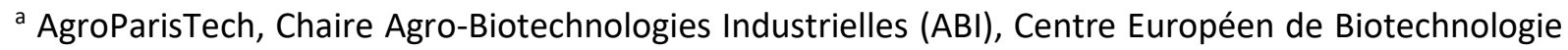 \\ et Bioéconomie (CEBB), 3 Rue des Rouges Terres, F-51110 Pomacle, France \\ b UMR GMPA, AgroParisTech, INRA, Université Paris-Saclay, F-78850, Thiverval-Grignon, France \\ * Corresponding author: marwen.moussa@agroparistech.fr
}

\section{Abstract:}

Reactive extraction is a well-known method for the recovery of organic acids from dilute streams. The extraction through complex formation with amines is followed by its back-extraction. Their affinity for amine extractants makes their back-extraction a limiting step. The case of 3-hydroxypropionic acid is addressed here. Three methods were compared: salts additions, diluent swing and temperature swing. Mineral bases led to complete 3-HP recovery but as a salt. Diluent swing was tested using $n$-hexane as the anti-solvent. Results showed that increasing the $n$-hexane content led to a great increase of 3-HP recovery. However, high recovery yields are associated with high dilutions. The temperature was varied from 4 to $140{ }^{\circ} \mathrm{C}$ and it led to a high decrease of the acid-amine complex formation. Our thermochemical study showed an apparent heat of reaction of $-25.3 \mathrm{~kJ} / \mathrm{mol}$ and, at $140{ }^{\circ} \mathrm{C}, 78 \%$ of 3HP can be recovered in a single step.

Keywords: back-extraction, $\mathrm{pH}$ swing, diluent swing, temperature swing 


\section{Introduction}

3-hydroxypropionic acid (aka 3-HP) is a hydrophilic organic acid that recently raised interest for the production of bio-based acrylate derivatives and polyesters [1]. It can be produced through the bioconversion of biomass-derived compounds like glucose and glycerol [2]. However, the resulting bioconversion broth can be quite diluted in 3-HP and contains water-soluble impurities. Reactive extraction is known as an efficient technique to selectively recover small organic acids from dilute aqueous phases [3]. In reactive extraction, the high selectivity and recovery yield are due to specific reactions involving a hydrophobic extractant dissolved in an organic phase and the acid present in the aqueous phase. Reactions lead to formation of hydrophobic complexes that are soluble in the organic phase. In the case of $3-\mathrm{HP}$, an organic phase made of $20 \% \mathrm{v} / \mathrm{v}$ tri- $n$-octylamine (TOA) in $n$-decanol has been proven successful for the acid removal from dilute aqueous media $(\sim 1 \mathrm{~g} / \mathrm{L})[4-6]$. Other extracting systems have been tested like TOA in other alcohols [4,7] or tributyl phosphate in heptane [7] but the latter was less efficient.

The reactive mechanism of 3-HP extraction by TOA in $n$-decanol is a favorable acid-base interaction that leads to the formation of an ion pair in the organic phase where 3-HP loses its acidic proton [8]:

$\mathrm{TOA}_{\text {org }}+A H_{\text {aq }} \rightleftharpoons\left(\mathrm{TOAH}^{+}, A^{-}\right)_{\text {org }}($ reaction 1)

Acid-amine complexes in organic phases are rather stable and the back-extraction of the acid into an aqueous phase can be relatively challenging. Several methods have been suggested in the literature for the back-extraction of organic acids from tertiary amines extracting systems: salt addition [9], acid displacement and distillation $[10,11], \mathrm{pH}$-swing (using mineral bases or water soluble amines) $[10,12,13]$, temperature swing $[14,15]$ and diluent swing $[16]$.

The addition of salts is mostly used for the stripping and regeneration of organic phases when the extractant used is a quaternary ammonium such as trioctylmethylammonium chloride [17] or Aliquat 336 [18] in an anion-exchange mode. However, it can also be used for tertiary amines. In an organic phase consisting in TOA diluted in chloroform, Puttemans et al [17] found that the back-extraction 
efficiency depended on the nature of the acid and on the concentration and type of anion of the sodium salts in the following order: perchlorate $\left(\mathrm{ClO}_{4}^{-}\right)>$iodide $\left(\mathrm{I}^{-}\right)>$bromide $\left(\mathrm{Br}^{-}\right)>$chloride $\left(\mathrm{Cl}^{-}\right)$. The salt has to be in large excess for a near complete recovery [17].

An original method is the displacement of the target acid from the organic phase to the aqueous phase by the use of another acid that has a better affinity for the extracting system. In this method, an aqueous phase loaded with such an acid in over-stoichiometry is used. For example, the use of hydrochloric acid in excess led to the recovery of $83 \%$ of the lactic acid present in an organic phase made of $30 \%$ Alamine 336 in oleyl alcohol [18]. In fact, hydrochloric acid is preferably extracted when compared to lactic acid and the extraction of $\mathrm{HCl}$ leads to the release of lactic acid under its nondissociated form in the aqueous phase. The yield of recovery of lactic acid depends on the concentration of $\mathrm{HCl}$. This latter needs to be set at the optimal level in order to prevent the presence of residual $\mathrm{HCl}$ in the back extraction aqueous phase. For a maximal recovery, $\mathrm{HCl}$ has to saturate the amine and in this case a substantial amount of $\mathrm{HCl}$ remains in water in mixture with lactic acid. Using less $\mathrm{HCl}$ leads to its complete extraction in the organic phase with no remaining amount in water, but the recovery of lactic acid is lower in this case. The amine loaded with $\mathrm{HCl}$ could be regenerated by distilling off $\mathrm{HCl}$. Acetic acid can also be used for this displacement method [10] and then it can be removed and recovered from the amine-containing organic phase by simple distillation [19].

A widely reported method for the recovery of organic acids from loaded organic phases is the use of basic water soluble compounds like sodium hydroxide and carbonate (basic mineral compounds) or trimethylamine (TMA, a weak base organic compound) $[10,12,13,20]$. In aqueous phase, these compounds impose a high pH that induces the release of the acid under its dissociated form. TMA or $n$-propylamine used in near stoichiometric amounts have been reported to be very efficient, giving a total acid recovery $[10,21]$. However, the process needs to be cautiously operated because such amines, if added in excess, can be extracted, at least in part, in the organic phase [10]. The recovery of the non-dissociated acid is made through the thermal decomposition of the ammonium salt thanks to 
the high volatility of the amine $[10,21]$. For instance, $99 \%$ of TMA could be removed from a mixture containing $0.25 \mathrm{~mol} / \mathrm{L}$ lactic acid and TMA in water under reduced pressure (200 mbar) and heating $\left(120^{\circ} \mathrm{C}\right)[22]$.

The temperature swing method relies on the thermal reversibility of the acid-amine complexation reaction [23]. Increasing the temperature of the organic phase in the presence of an aqueous phase can lead to the dissociation of the complex into its two components: the acid in the aqueous phase and the amine in the organic phase. Therefore, heating a loaded organic phase in contact with an aqueous phase can lead to some back-extraction. This method is attractive as it does not need the addition of any chemicals and allows the recovery of the protonated acid [12,23-27].

The diluent swing method consists in adding a poorly solvating diluent, called here "anti-solvent", to the organic phase of extraction [23]. It is well known that reactive extraction efficiency depends on the solvating properties of the diluent with alcohols being regularly the best solvents. If the so-called antisolvent is added to a loaded organic phase, then the resulting phase will have lower extraction ability $[28,29]$ and reject the acid in the aqueous phase, leading to a back-extraction effect $[12,23]$. This organic phase can then be regenerated, for example by distilling off the anti-solvent (if more volatile) which is then recycled [23].

3-HP is a relatively recent subject of interest and only few studies dealt with its reactive extraction and none with its back-extraction. In our previous publications, we presented results concerning 3-HP reactive extraction and its mechanisms[4,8]. To the best of our knowledge, this work is the first experimental study concerning 3-HP back-extraction. This paper addresses the back-extraction of 3hydroxypropionic acid from loaded organic phases of 20\%v/v TOA in $n$-decanol focusing on bases and salts addition, diluent swing and temperature swing. The implications of the involved mechanisms on process design are discussed. In the case of sodium hydroxide, an example is provided where the backextraction is performed with a membrane contactor, a non-dispersive liquid-liquid contactor avoiding emulsions and widely studied in biotechnological applications. 


\section{Material and Methods}

\subsection{Chemicals}

Organic phases were made of $n$-decanol ( $99 \%$ purity) and tri- $n$-octylamine ( $98 \%$ purity) from SigmaAldrich (USA). For some experiments, n-hexane ( $>98 \%$ purity, Fischer, USA) was added. 3hydroxypropionic acid was purchased in $30 \%$ wt solution in water (TCI Europe, Belgium). Saline solutions consisted in sodium chloride $(\mathrm{NaCl}, 100 \%$ purity), sodium hydroxide ( $\mathrm{NaOH},>99 \%$ purity) and sodium carbonate $\left(\mathrm{Na}_{2} \mathrm{CO}_{3},>99 \%\right.$ purity) from VWR (USA).

\subsection{Experiments}

All organic phases were initially made of $20 \% \mathrm{v} / \mathrm{v}$ TOA in decanol. Organic phases were first loaded with 3-HP by reactive liquid-liquid extractions as described previously [4]; they were therefore saturated with water. Back-extraction of such loaded organic phases was performed using salt addition, diluent swing and temperature swing. Except for one experiment described below, the temperature was always controlled using a laboratory oven.

\subsubsection{Back-extraction using salts additions}

Equilibrium study using tests in tubes: An aqueous phase $(10 \mathrm{~mL})$ containing the salt was mixed into a centrifuge tube with an equal volume of organic phase loaded with $3-\mathrm{HP}(1 \mathrm{~g} / \mathrm{L})$. The centrifuge tube was then manually shaken for 3 minutes and left overnight at $25^{\circ} \mathrm{C}$ before being manually shaken again for 3 minutes and centrifuged at $15557 \mathrm{~g}$ for 20 minutes.

Kinetic study using membrane contactor: An example of back-extraction with $\mathrm{NaOH}$ in stoichiometric proportion using an organic phase loaded with $3-\mathrm{HP}(8 \mathrm{~g} / \mathrm{L})$ was performed using a LiquiCel $2.5 \times 8$ membrane contactor with X50 polypropylene fibers (40\% porosity) and the back-extraction kinetics was compared to the extraction one. Each phase had a constant volume of $500 \mathrm{~mL}$ and were continuously stirred and set to $25^{\circ} \mathrm{C}$ using a heated water bath. The aqueous phase was pumped through the lumen side of the fibers at a flowrate of $8.6 \mathrm{~mL} / \mathrm{s}$ and the organic phase through the shell side of the module at a flowrate of $8.1 \mathrm{~mL} / \mathrm{s}$ in counter-current cross-flow configuration. In such a 
configuration, the liquid-liquid interface lies at the internal surface of the fibers $\left(1 \mathrm{~m}^{2}\right)$ and the flowrates are high enough so that the hydrodynamics do not control the transfer rate [30]. It has been made sure by increasing the flowrates and checking that the transfer rates were not influenced.

\subsubsection{Diluent swing back-extraction}

Hexane was used as the anti-solvent. It was added to organic phases loaded with 3-HP (4.4 g/L) in order to represent 20,40 and $60 \%$ of the final volume. $10 \mathrm{~mL}$ of the resulting organic phases were obtained in centrifuge tubes. A diffuse white cloud immediately appeared due to a small fraction of soluble water that was rejected from the organic phase because of the presence of hexane. Ultrapure water was then added into each tube to recover the 3-HP. Three aqueous/organic volume ratios were used (Table 1): a ratio where the water volume corresponds to the half of the organic phase without hexane (ratio 1), a ratio where the water volume was equal to the one of the organic phase without hexane (ratio 2) and a ratio where the water volume was equal to the one of the final organic phase containing hexane (ratio 3). The tubes were then manually shaken for 3 minutes and left overnight at $25^{\circ} \mathrm{C}$. They were then manually shaken again for 3 minutes and centrifuged at $15557 \mathrm{~g}$ for 10 minutes.

Table 1: Loaded organic phase, hexane and water volumes used in solvent swing experiments

$\begin{array}{cccc}\text { 20\% } n \text {-hexane } & \text { Ratio } 1 & \text { Ratio } 2 & \text { Ratio } 3 \\ \text { TOA/decanol with 3-HP } & 8 \mathrm{~mL} & 8 \mathrm{~mL} & 8 \mathrm{~mL} \\ n \text {-hexane } & 2 \mathrm{~mL} & 2 \mathrm{~mL} & 2 \mathrm{~mL} \\ \text { water } & 4 \mathrm{~mL} & 8 \mathrm{~mL} & 10 \mathrm{~mL} \\ 40 \% \text { v/v } n \text {-hexane } & \text { Ratio } 1 & \text { Ratio } 2 & \text { Ratio } 3 \\ \text { TOA/decanol with 3-HP } & 6 \mathrm{~mL} & 6 \mathrm{~mL} & 6 \mathrm{~mL} \\ n \text {-hexane } & 4 \mathrm{~mL} & 4 \mathrm{~mL} & 4 \mathrm{~mL} \\ \text { water } & 3 \mathrm{~mL} & 6 \mathrm{~mL} & 10 \mathrm{~mL} \\ 60 \% \text { v/v } n \text {-hexane } & \text { Ratio } 1 & \text { Ratio } 2 & \text { Ratio } 3 \\ \text { TOA/decanol with 3-HP } & 4 \mathrm{~mL} & 4 \mathrm{~mL} & 4 \mathrm{~mL} \\ n \text {-hexane } & 6 \mathrm{~mL} & 6 \mathrm{~mL} & 6 \mathrm{~mL} \\ \text { water } & 2 \mathrm{~mL} & 4 \mathrm{~mL} & 10 \mathrm{~mL}\end{array}$

\subsubsection{Temperature swing back-extraction}

Aqueous solutions containing 3-HP at $6,11,16,21$ and $26 \mathrm{~g} / \mathrm{L}$ were prepared. 3-HP extraction was performed at $4,37,60,90$ and $140^{\circ} \mathrm{C} .8 \mathrm{~mL}$ of each aqueous phase were placed in centrifuge tubes containing $8 \mathrm{~mL}$ of organic phase not loaded with 3-HP. The tubes were then placed to equilibrate at 
$4{ }^{\circ} \mathrm{C}$ in a cold-storage chamber for $30 \mathrm{~min}$ and then shaken and placed back for an hour at $4{ }^{\circ} \mathrm{C}$. The tubes were then centrifuged at $4^{\circ} \mathrm{C}$ before sampling $1 \mathrm{~mL}$ of the organic phase and $1 \mathrm{~mL}$ of the aqueous phase for analysis. The tubes were then shaken and placed in a laboratory oven at $37^{\circ} \mathrm{C}$ for $30 \mathrm{~min}$, shaken again and let to equilibrate for an hour at $37^{\circ} \mathrm{C}$ before centrifugation for 10 minutes at $37^{\circ} \mathrm{C}$. Samples of $1 \mathrm{~mL}$ were then taken from the organic and aqueous phases for analysis. Similar procedures were performed for 60 and $90{ }^{\circ} \mathrm{C}$. The temperature was checked before each sampling. For the extraction at $140{ }^{\circ} \mathrm{C}$, the procedure was different. 3-HP solutions were used $(5,10,14$ and $19 \mathrm{~g} / \mathrm{L})$ and $8 \mathrm{~mL}$ of each solution were placed in centrifuge tubes containing $8 \mathrm{~mL}$ of a fresh organic phase. The tubes were shaken and placed in a bath of heat transfer oil regulated at $140{ }^{\circ} \mathrm{C}$ for $10 \mathrm{~min}$, then shaken again and placed back. After $1 \mathrm{~h}$, the tubes were directly quenched in melting ice and an aqueous sample was immediately taken at the bottom of the tube using a syringe.

\subsection{Analytical methods}

3-HP concentrations in aqueous phases were determined with high performance liquid chromatography (HPLC) using a method detailed previously[4]. The overall analysis uncertainty, defined as the coefficient of variation of the internal standard peak area, was evaluated to $1 \%$. The amount of 3-HP in the organic phase was first determined by mass balance knowing the amount of 3HP introduced initially for the extractions dedicated to the loading of organic phases. Then, in the light of the results (section 3.1.), back-extraction using $\mathrm{NaOH}$ solutions was used and these solutions were analyzed using HPLC. The validity of this method with back-extraction will be checked in the results section.

Experiments were at least performed in duplicate.

\section{Parameters definition}

The recovery yield $(Y)$ represents the proportion of acid back-extracted from the organic phase. As phase volume ratios were found to be constant during experiments, the recovery yield was calculated as follows: 


$$
Y=\frac{\left[A H_{a q, T O T}\right]^{e q} \times V_{a q}}{\left[A H_{\text {org }, \text { TOT }}\right]^{\text {ini }} \times V_{\text {org }}}
$$

Where $\left[A H_{a q, T O T}\right]^{e q}$ is the total acid concentration under all forms in the aqueous phase at equilibrium and $\left[A H_{\text {org,TOT }}\right]^{\text {ini }}$ is the total acid concentration under all forms in the organic phase initially.

In order to discriminate the effect of temperature between physical (i.e. without TOA) and reactive extraction, some extractions were performed using an organic phase only made of decanol. The partition coefficient $(\mathrm{m})$ for each temperature is defined as:

$$
m=\frac{\left[A H_{\text {org }}\right]^{e q}}{\left[A H_{a q}\right]^{e q}}
$$

Where $\left[\mathrm{AH} \mathrm{org}^{e q}\right.$ is the concentration of the free protonated acid in the organic phase at equilibrium and $\left[A H_{a q}\right]^{e q}$ the concentration of the free protonated acid in the aqueous phase at equilibrium. And it was calculated as the slope of the linear regression plot of the organic equilibrium concentrations against the aqueous equilibrium concentrations.

The complexation equilibrium constant between TOA and 3-HP is defined by the following ratio (reaction 1):

$$
K_{11}=\frac{\left[\left(\mathrm{TOAH}^{+}, A^{-}\right)_{\text {org }}\right]^{e q}}{\left[A H_{\text {aq }}\right]^{e q}\left[\mathrm{TOA}_{\text {org }}\right]^{e q}}
$$

Where $\left[\left(\mathrm{TOAH}^{+}, A^{-}\right)_{\text {org }}\right]^{e q}$ is the acid-amine complex concentration in the organic phase at equilibrium and $\left[\mathrm{TOA}_{\text {org }}\right]^{e q}$ the TOA concentration in the organic phase at equilibrium.

The kinetics in membrane contactor were evaluated using the $t_{63 \%}$, the time needed to reach $63 \%$ of the total extracted or back-extracted 3-HP. Parameter $t_{63 \%}$ would correspond to a time constant if extraction and back-extraction followed first-order kinetics.

The distribution coefficient is often found in the literature and is defined as the following ratio:

$$
D=\frac{\left[A H_{\text {org }, T O T}\right]^{e q}}{\left[A H_{\text {aq,TOT }}\right]^{e q}}
$$


Where $\left[A H_{\text {org,TоT }}\right]^{e q}$ is the total acid concentration under all forms in the organic phase at equilibrium.

\section{Results and discussion}

\subsection{Back-extraction using salts addition}

\subsubsection{Equilibrium study}

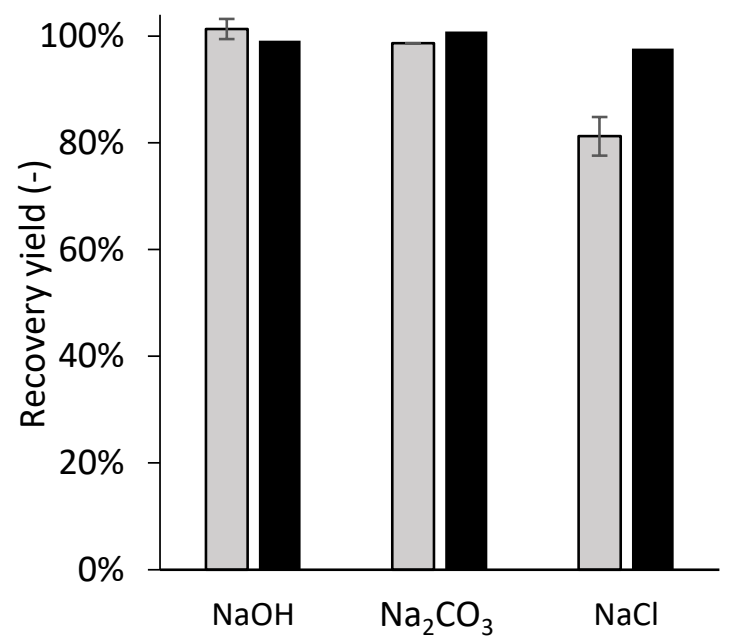

Figure 1: Recovery yields for back-extraction experiments using salts in stoichiometric proportions ( ) or 20-fold excess of salt ( ) at $25^{\circ} \mathrm{C}$ for $1 \mathrm{~g} / \mathrm{L}$ of 3-HP initially in the organic phase

Two bases $\left(\mathrm{NaOH}\right.$ and $\left.\mathrm{Na}_{2} \mathrm{CO}_{3}\right)$ and one salt $(\mathrm{NaCl})$ were tested. Two concentration levels were tested for each salt: stoichiometric concentration according to the 3-HP amount (11 mM; $1 \mathrm{~g} / \mathrm{L})$ in the organic phase and 20-fold excess. Note that for $\mathrm{Na}_{2} \mathrm{CO}_{3}$, the stoichiometric condition means half the amount of 3-HP because of its dibasic property. For each salt and base in particular:

- $\mathrm{NaOH}: 11 \mathrm{mM}(0.44 \mathrm{~g} / \mathrm{L})$ and $220 \mathrm{mM}(8.8 \mathrm{~g} / \mathrm{L})$

- $\mathrm{NaCl}: 11 \mathrm{mM}(0.64 \mathrm{~g} / \mathrm{L})$ and $220 \mathrm{mM}(12.8 \mathrm{~g} / \mathrm{L})$

- $\quad \mathrm{Na}_{2} \mathrm{CO}_{3}: 5.5 \mathrm{mM}(0.58 \mathrm{~g} / \mathrm{L})$ and $110 \mathrm{mM}(11.7 \mathrm{~g} / \mathrm{L})$

Figure 1 shows that for all the species, it has been possible to recover nearly all the 3-HP present initially in the organic phase ( $\geq 98 \%$ ) depending on the species concentration. For $\mathrm{NaOH}$ and $\mathrm{Na}_{2} \mathrm{CO}_{3}$, stoichiometric proportions were enough to reach this recovery yield, while for $\mathrm{NaCl}$ an excess stoichiometry was necessary. For the bases, a quantitative reaction of neutralization takes place given the $\mathrm{pK}_{\mathrm{A}}$ difference between 3-HP $\left(p K_{A}^{3-H P}=4.5\right)$ and the bases $\left(p K_{A}^{\mathrm{H}_{2} \mathrm{O} / \mathrm{HO}^{-}}=14, p K_{A}^{\mathrm{CO}_{2}, \mathrm{H}_{2} \mathrm{O} / \mathrm{HCO}_{3}^{-}}=\right.$ 
6.4, $\mathrm{pK}_{\mathrm{A}}^{\mathrm{HCO}_{3}^{-} / \mathrm{CO}_{3}^{2-}}=10.3$ ) leading to quantitative 3- $\mathrm{HP}$ recovery in stoichiometric proportions as the dissociated form of 3-HP is not soluble in the organic phase. The overall reactions can be written as follows in the case of $\mathrm{NaOH}$ :

$\left(\mathrm{TOAH}^{+}, A^{-}\right)_{\text {org }} \rightleftharpoons \mathrm{TOA}_{\text {org }}+A H_{\text {aq }}$ (reaction 2, unfavorable)

$\mathrm{AH}_{a q}+\mathrm{HO}_{a q}^{-} \rightleftharpoons A_{a q}^{-}+\mathrm{H}_{2} \mathrm{O}$ (reaction 3, quantitative)

$\rightarrow\left(\mathrm{TOAH}^{+}, \mathrm{A}^{-}\right)_{\text {org }}+\mathrm{HO}_{a q}^{-} \rightleftharpoons \mathrm{TOA}_{\text {org }}+A_{a q}^{-}+\mathrm{H}_{2} \mathrm{O}$ (reaction 4, quantitative)

According to this mechanism the amine extractant is regenerated and can be recycled. Since reaction 4 is quantitative with a $100 \%$ recovery, back-extraction using $\mathrm{NaOH}$ can be used to determine the amount of 3-HP in the organic phase, validating this method for further analyses.

In the case of $\mathrm{Na}_{2} \mathrm{CO}_{3}$, the overall reactions can be written as follows:

$\mathrm{AH}_{a q}+\mathrm{CO}_{3}^{2-}{ }_{a q} \rightleftharpoons A_{a q}^{-}+\mathrm{HCO}_{3}^{-}{ }_{a q}$ (reaction 5, quantitative)

$\mathrm{AH}_{a q}+\mathrm{HCO}_{3}^{-}{ }_{a q} \rightleftharpoons A_{a q}^{-}+\mathrm{CO}_{2 a q}+\mathrm{H}_{2} \mathrm{O}$ (reaction 6, favorable)

$\mathrm{CO}_{2 a q} \rightleftharpoons \mathrm{CO}_{2 g}$ (reaction 7, open to air)

$\rightarrow 2\left(\mathrm{TOAH}^{+}, \mathrm{A}^{-}\right)_{\text {org }}+\mathrm{CO}_{3}^{2-}{ }_{a q} \rightleftharpoons 2 \mathrm{TOA}_{\text {org }}+2 \mathrm{~A}_{\text {aq }}^{-}+\mathrm{CO}_{2 g}+\mathrm{H}_{2} \mathrm{O}$ (reaction 8, quantitative)

This mechanism is supported by the gas release observed when the lid was opened after reaching equilibrium due to $\mathrm{CO}_{2}$ degassing from water. The release of $\mathrm{CO}_{2}$ makes it possible to regenerate the amine extractant in the organic phase allowing the organic phase to be recycled, as seen for $\mathrm{NaOH}$. However, the acid is recovered under its sodium salt form. Moreover, a washing of the organic phase after the back-extraction may be necessary to remove traces of the inorganic base. A simplified process diagram is proposed in Figure 2 for illustration purposes in the case of back-extraction using sodium hydroxide. 


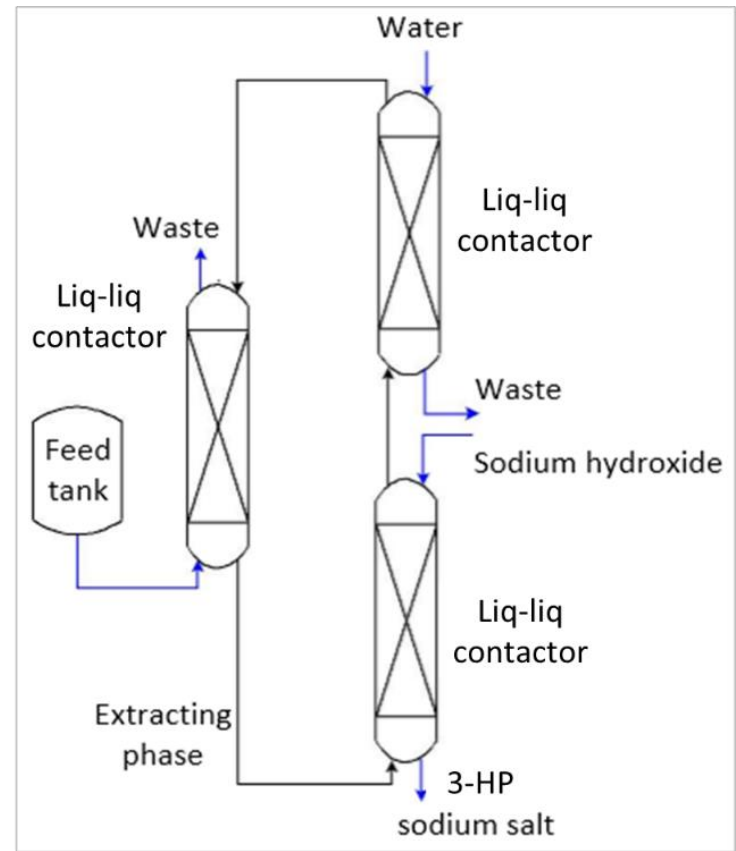

Figure 2: Simplified process diagram of extraction and back-extraction using sodium hydroxide

When sodium chloride is used, a quantity similar to the one of 3-HP in the organic phase is not enough to entirely back-extract the $3-\mathrm{HP}(\mathrm{Y}=80 \%$, Figure 1$)$. Indeed, the mechanism involved in this case is an anion exchange reaction (reaction 9).

$\left(\mathrm{TOAH}^{+}, \mathrm{A}^{-}\right)_{\mathrm{org}}+\mathrm{Cl}_{a q}^{-} \rightleftharpoons\left(\mathrm{TOAH}^{+}, \mathrm{Cl}^{-}\right)_{\mathrm{org}}+A_{a q}^{-}$(reaction 9, favorable)

Such reactions are governed by chemical equilibria, which means that an excess of reactant is necessary to reach a nearly complete conversion [17]. Besides this drawback, using salts without basic properties does not regenerate the amine which remains protonated, i.e. unable to bind again to acids. Moreover, the acid is recovered under its deprotonated form. Therefore, these salts are not recommanded.

\subsubsection{Study of back-extraction kinetics in a membrane contactor using $\mathrm{NaOH}$}




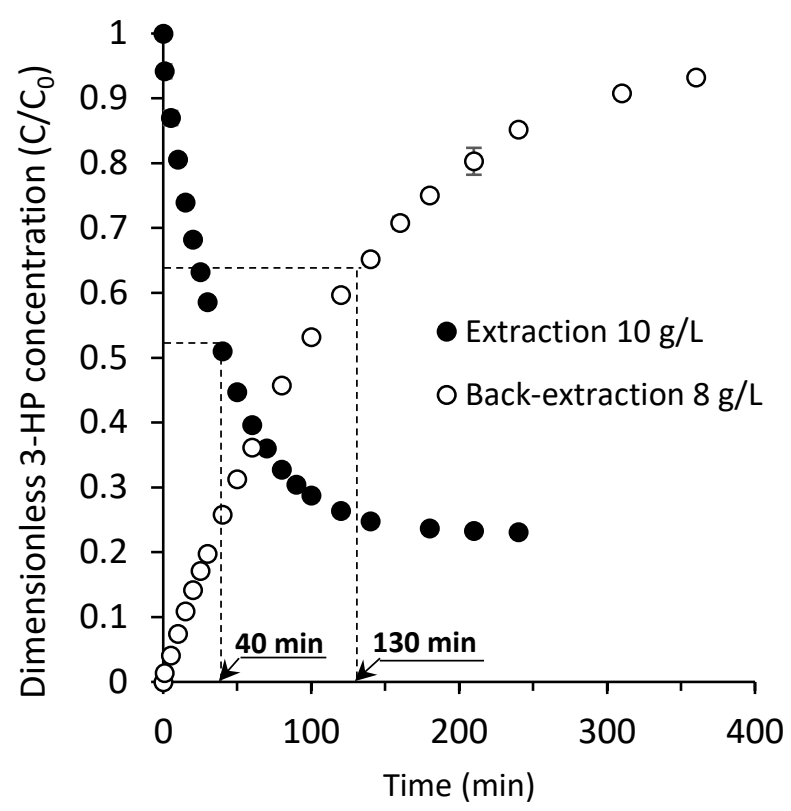

Figure 3: Evolution of 3-HP concentration in the aqueous phase during extraction and back-extraction $(10 \mathrm{~g} / \mathrm{L} \mathrm{3-HP}$ in the aqueous phase and $8 \mathrm{~g} / \mathrm{L} 3-\mathrm{HP}$ in the organic phase initially for extraction and backextraction respectively)

Aqueous solution of $\mathrm{NaOH}$ in stoichiometric proportion was used for the membrane-based backextraction of 3-HP from an organic phase composed of $20 \%$ TOA in decanol. The evolution of 3-HP concentration in the back-extraction aqueous phase is plotted against time in figure 3 (open circles). For the sake of comparison, the evolution of 3-HP concentration for the case of a forward extraction from the aqueous to the organic phase is also plotted on the same figure (filled circles). It can be seen that back-extraction takes more than $6 \mathrm{~h}$ to complete while the forward extraction reaches equilibrium within 3-4h. Accordingly, the back-extraction kinetics is much slower than the extraction one, as shown by the time needed to reach $63 \%$ of the equilibrium value: $40 \mathrm{~min}$ for extraction, $130 \mathrm{~min}$ for backextraction. This behavior is somehow unexpected as the back-extraction yield is total, higher than the extraction yield (77\%). Indeed, it should be reminded that most of the mass transfer resistance is expected to be located in the organic phase and in particular in the membrane pores filled with the organic phase which is much more viscous than the aqueous phase. Usual expressions of the mass flux in the membrane pores expressed with a mass transfer coefficient (k), a surface area (S) and a driving force (concentration difference) in the case of the forward and back extraction are:

$J_{F E}=k \cdot S .\left(C_{\text {int }}^{F E}-C_{\text {org }}^{F E}\right)$ for the forward extraction (FE) 
$J_{B E}=k \cdot S \cdot\left(C_{\text {org }}^{B E}-C_{\text {int }}^{B E}\right)$ for the back extraction (BE)

Where here and further in the text:

$C_{i n t}$ concentration at the liquid-liquid interface, organic side (in the membrane pores at the interface)

$C_{\text {org }}$ concentration in the organic bulk (other extremity of the pores towards the bulk)

$C_{a q}$ concentration in the aqueous bulk

In the case of forward extraction, at the beginning, organic interfacial concentration of 3-HP $\left(C_{\text {int }}^{F E}\right)$ can be high due to favorable instantaneous complexation equilibrium $\left(C_{i n t}^{F E}>C_{a q}^{F E}\right)$ while in the organic bulk $\left(C_{\text {org }}^{F E}\right)$ the initial concentration is 0 so that the maximum driving force in the membrane pores is high. Conversely, for the back-extraction, at the beginning, the organic interfacial concentration $\left(C_{i n t}^{B E}\right)$ is close to 0 as the reaction between $3-\mathrm{HP}$ and hydroxide ions is total and instantaneous and the concentration of 3-HP in the pores is kept low. The maximum driving force through the membrane pores is then limited by the initial concentration of 3-HP in the organic phase $\left(C_{\text {org }}^{B E}-C_{\text {int }}^{B E}=C_{\text {org }}^{B E}\right)$ and then initially we have $\left|C_{\text {int }}^{F E}-C_{\text {org }}^{F E}\right|=C_{\text {int }}^{F E}>\left|C_{\text {org }}^{B E}-C_{\text {int }}^{B E}\right|=C_{\text {org }}^{B E}$. This explains why back-extraction is so slow compared to the extraction. Such a behavior should be taken into consideration for an optimized design of continuous processes including integrated forward and back-extraction steps.

\subsection{Back-extraction using diluent swing}
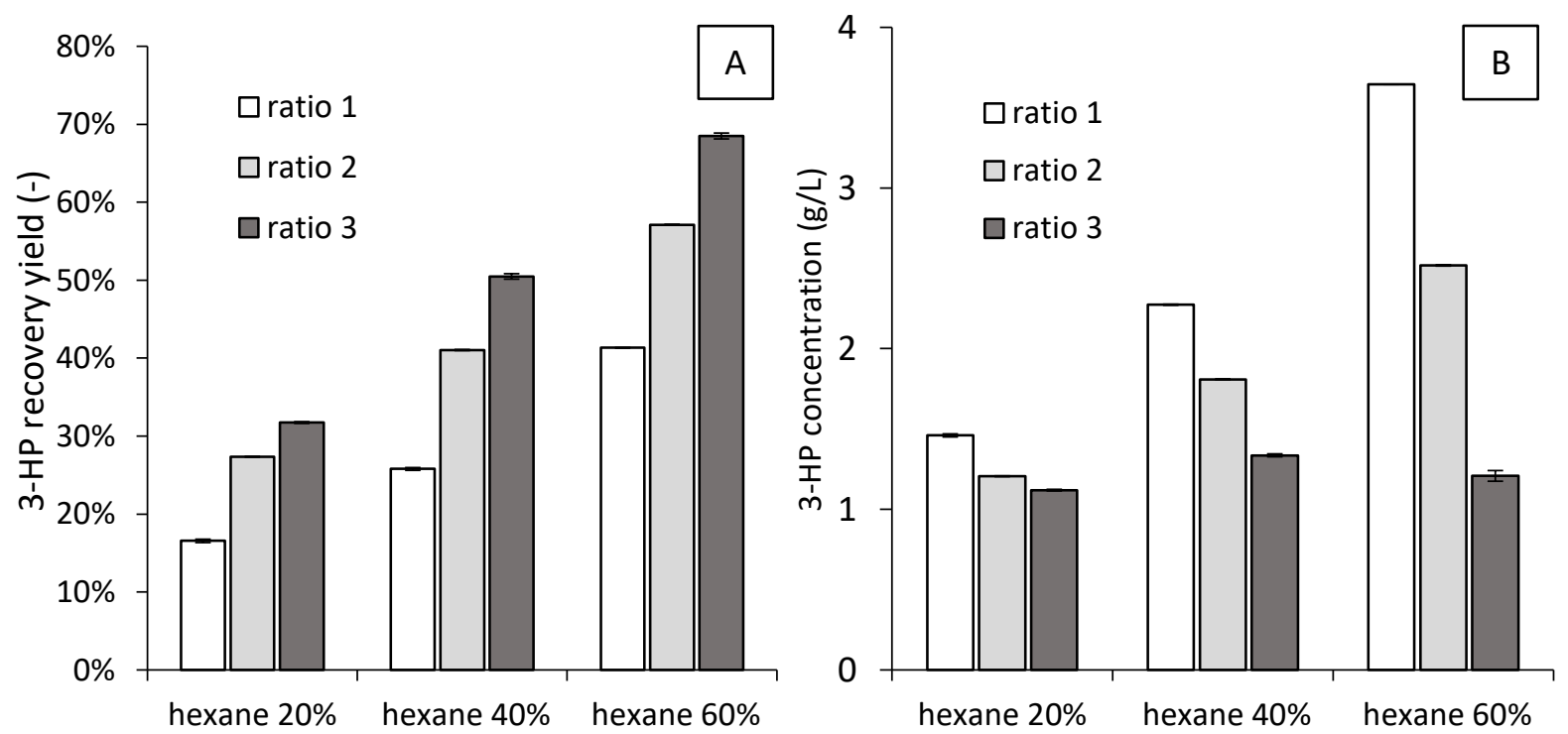

Figure 4: recovery yield (A) and 3-HP concentration in the recovery phase (B) after diluent swing backextraction as a function of the volume fractions of hexane and aqueous/organic volume ratios. 
Hexane was used for diluent swing as it is known to have poor extraction ability towards 3-HP with TOA [4]. Another interesting fact about hexane is its high volatility and easy regeneration by distillation. Nine experimental conditions were tested varying both the hexane content and the volume ratio between the organic and the aqueous phase of back-extraction. Results are shown in figure 4. For a given volume ratio, it can be seen that increasing the amount of hexane increases the back-extraction yield, as the ability of the organic phase to stabilize the 3-HP-TOA complex is decreased. Hence, 3-HP is more rejected into the back-extraction aqueous phase. Concerning the volume ratio, increasing the volume of water (ratio 3 vs ratio 1 ) increases the recovery yield but decreases the final 3-HP concentration in the aqueous phase. For example, for $60 \% \mathrm{v} / \mathrm{v}$ hexane, ratio 1 (lowest aqueous/organic ratio, see section 2.2 .2 .) leads to a concentration in the recovery phase corresponding to $83 \%$ of that in the initial organic phase $(4.4 \mathrm{~g} / \mathrm{L})$ but the recovery yield is only $41 \%$. Conversely, the ratio 3 (highest aqueous/organic ratio) leads to a concentration in the recovery phase corresponding to $28 \%$ of that in the initial organic phase but the recovery yield is quite high at almost $70 \%$. In comparison, diluent swing with a TOA/decanol/hexane mixture has been reported to be much less efficient for propionic acid [12]. For example, addition of $50 \% \mathrm{v} / \mathrm{v}$ hexane using ratio 3 for back-extraction resulted in only 8 $9 \%$ of propionic acid recovery. This is probably due to the much higher hydrophobicity of propionic acid compared to 3-HP due to the absence of the hydroxyl group, making it less sensitive to the solvent mixture polarity.

Hexane is a volatile solvent (low boiling point and molar heat of vaporization) and therefore can easily be distilled off from the organic phase at rather low temperature. For example, the bubble point of mixtures containing 20 to $60 \% \mathrm{v} / \mathrm{v}$ hexane in TOA/decanol is predicted to range roughly between 120 and $80{ }^{\circ} \mathrm{C}$, with a vapor phase made of more than $99 \%$ mol hexane (UNIFAC 1993 calculation, data not shown). A possible simplified flow diagram of the process is proposed in Figure 5. 


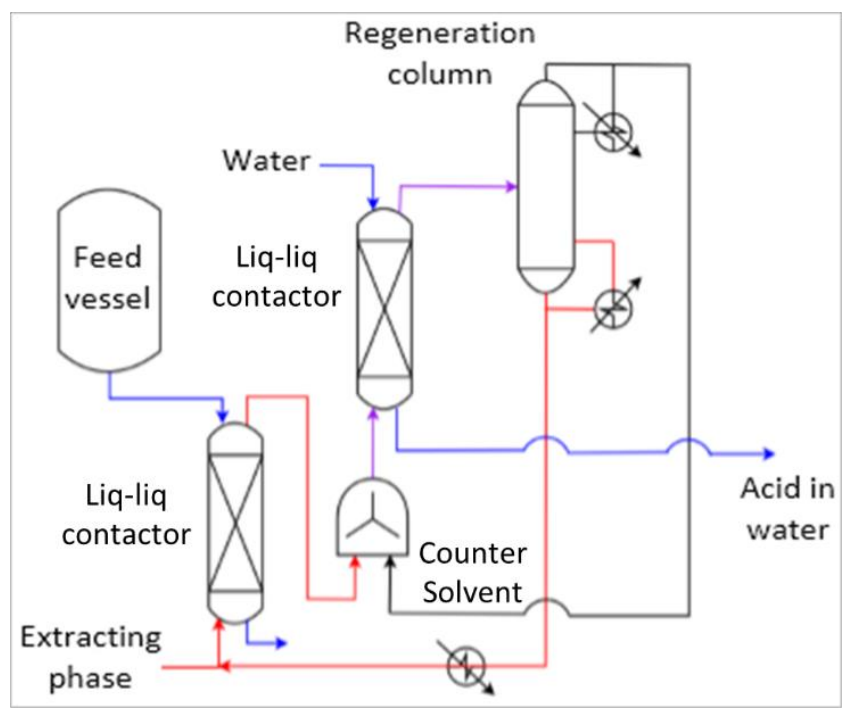

Figure 5: Simplified process diagram of extraction and back-extraction using diluent swing with a volatile anti-solvent such as hexane.

\subsection{Back-extraction using temperature swing}

Temperature swing states that, after extraction, the acid is recovered in the aqueous phase upon heating. It is meant to take advantage of the complexation reaction reversibility upon heating. In order to evaluate the extent of this phenomenon, we first studied the influence of temperature on the extraction efficiency. Figure 6 shows the extraction yields as a function of the initial 3-HP concentration and extraction temperature.
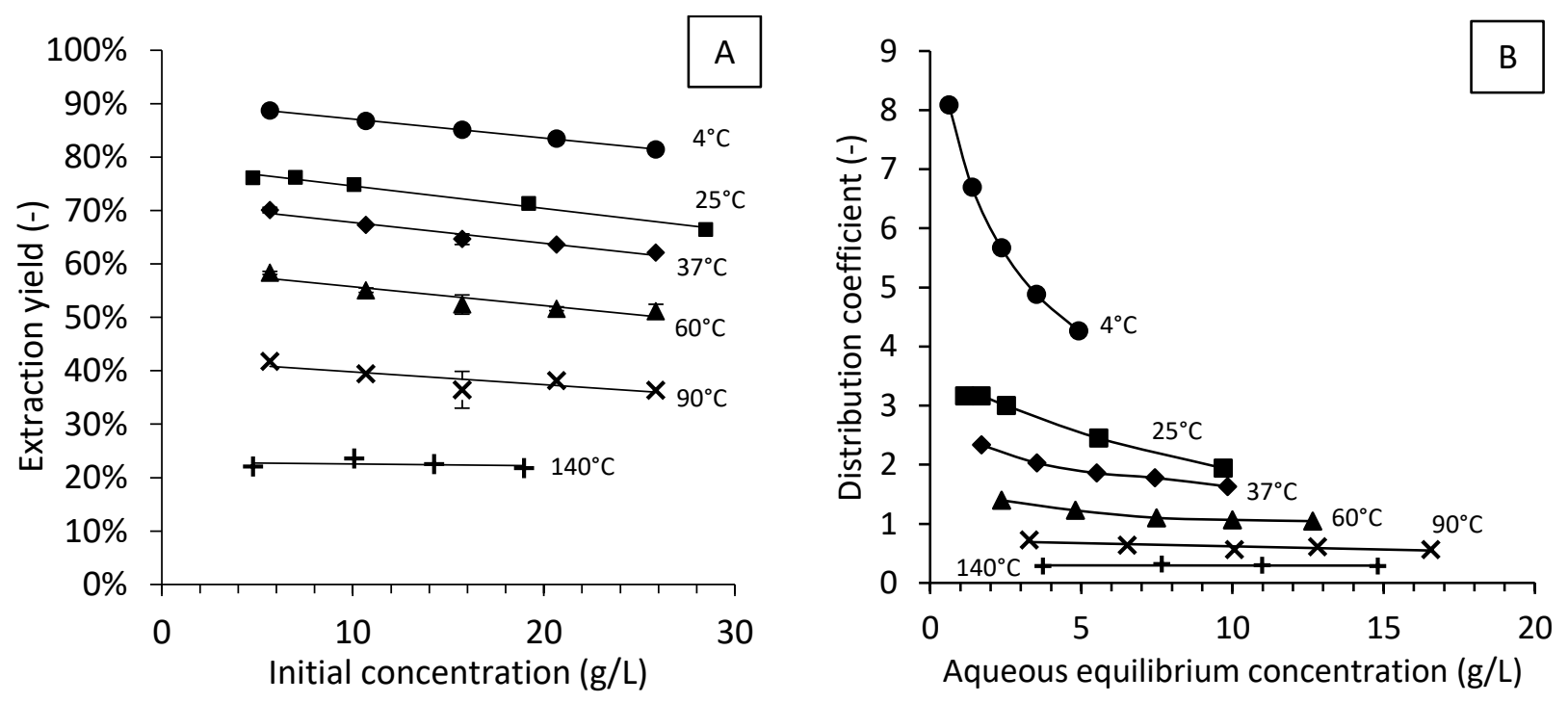

Figure 6: (A) extraction yield as a function of the initial 3-HP concentration in the aqueous phase and extraction temperature, (B) distribution coefficient as a function of the equilibrium concentration of 3$H P$ in the aqueous phase and extraction temperature (solid lines for clarity purpose only) 
It can be seen from figure 6 that the extraction yield strongly depends on temperature. For the same acid concentrations the higher the temperature, the lower the yield. For example, for 5-6 g/L of initial acid concentration, the extraction yield drops from 89 to $22 \%$ between 4 and $140{ }^{\circ} \mathrm{C}$. At $90{ }^{\circ} \mathrm{C}$ for example, the extraction yield is only about $40 \%$ meaning that the back-extraction would yield around $60 \%$. These results are beyond what has been reported for propionic acid at $90{ }^{\circ} \mathrm{C}(25-35 \%)$ [12] or lactic acid at $80^{\circ} \mathrm{C}$ (32\%) [28] with TOA in decanol as the organic phase. The effect of temperature was also studied in the case of physical extraction, i.e. with only decanol in the organic phase, showing that the extraction yield increases with temperature (ranging from 2 to $6 \%$ between 25 and $90{ }^{\circ} \mathrm{C}$; data not shown).

The overall thermodynamic pathway for the global transfer reaction phenomenon can be summarized as follows [23]: (i) heating or cooling the reacting species in solution from their actual temperature at their equilibrium concentration to $25^{\circ} \mathrm{C}$ (standard state), (ii) eliminating interactions between those species (heat of mixing) in order to consider pure reactants (standard state), (iii) reaction of the pure species at $25^{\circ} \mathrm{C}$ to form pure products (heat of reaction), (iv) mixing of all the species back together in solution (heat of mixing) at their equilibrium concentrations, $(v)$ heating or cooling the solution from $25^{\circ} \mathrm{C}$ to their actual temperature. Steps (ii) and (iv) represent non-idealities in composition change and steps (i) and (v) non-idealities in temperature change due to variations in physicochemical interactions, i.e. in species activity. As we do not discriminate between all these phenomena, the thermodynamic properties for the global extraction are called apparent.

Based on our previous results [4], we know that the complexation product, which is insoluble in the aqueous phase, is formed in the organic phase thanks to diluent solvation in particular through dipolar interactions and $\mathrm{H}$-bonding. When the temperature increases, the dielectric constant of $n$-alcohols decreases as well [31,32] (from 8.1 to 6.0 between 20 and $60{ }^{\circ} \mathrm{C}$ for $n$-decanol, from 14.1 to 5.8 between 0 and $100{ }^{\circ} \mathrm{C}$ for $n$-heptanol [33]). This indicates a decrease in their bulk polarity and a weaker $\mathrm{H}$-bonding effects. The thermal energy and randomness increase with temperature, reducing dipole 
organizations and the stabilizing effects of $\mathrm{H}$-bonds which may break more easily [31,34]. This explains why the overall reactive extraction yield decreases strongly with the increase of temperature and it can be described as follows.

First, we have to consider that the acid exists in the organic phase under two forms (free and bound to the amine) and that the transfer from the aqueous to the organic phase is made through 2 main mechanisms: (1) a physical transfer due to the acid solubility in the solvent and (2) a chemical transfer due to the complexation reaction with TOA in the organic phase. To discriminate between these two mechanisms, the physical extraction is studied first. To this end, a series of extractions were performed at different temperatures to determine the temperature dependence of the physical partition of 3-HP between water and $n$-decanol.

From the Van't Hoff equation and assuming that the global apparent enthalpy and entropy changes of reaction are constant over the temperature range tested, the following linear form can be derived:

$$
\ln (m)=-\frac{\Delta_{r} H}{R T}+\frac{\Delta_{r} S}{R}
$$

Where $\Delta_{r} H$ is the apparent molar enthalpy change of reaction (also called heat of reaction), $\Delta_{r} S$ is the molar entropy change of reaction, $R$ is the ideal gas constant and $T$ is temperature.

The corresponding linear regression for the partition coefficient $m$ is provided in the following Van't Hoff plot (figure 7), allowing to determine the related thermochemical parameters.

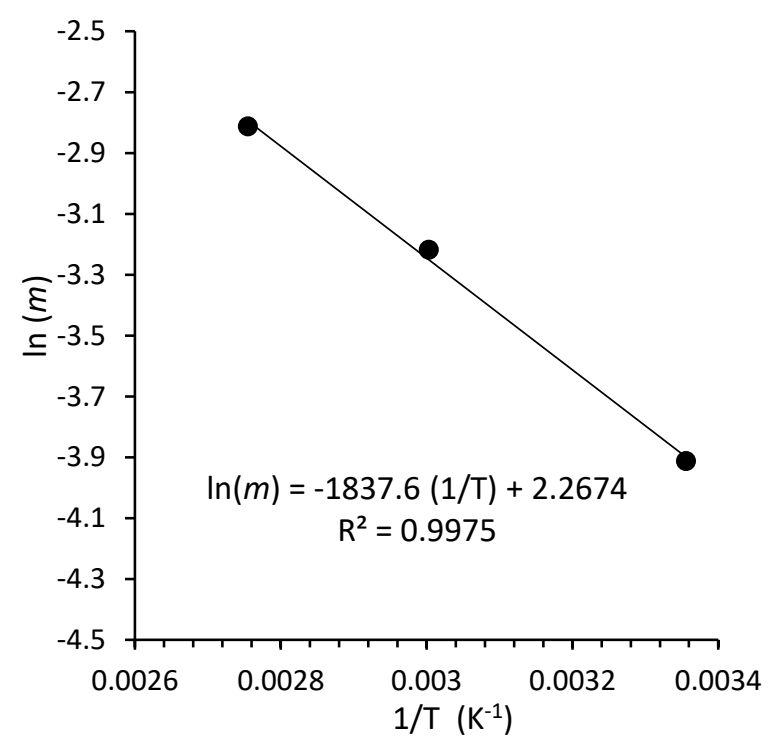

Figure 7: Van't Hoff plot of the physical partition coefficient (m) of 3-HP between water and $n$-decanol 
The 3-HP transfer from water to $n$-decanol causes an apparent enthalpy change of $15.3 \mathrm{~kJ} \cdot \mathrm{mol}^{-1}$ and an apparent entropy change of $18.9 \mathrm{~J} \cdot \mathrm{mol}^{-1} \cdot \mathrm{K}^{-1}$. This positive enthalpy change indicates the transfer is not energetically favorable, probably due to the lesser extent of solvation of 3-HP by $n$-decanol than water. The entropy change is also positive which can be explained by the fact that $3-\mathrm{HP}$ is partitioned in a greater volume with more physical states of solvation. Accordingly, the physical transfer is an entropy-driven process with less stable 3-HP in the organic phase.

As the variation of physical extraction with temperature is known, it is then possible to consider the temperature dependence of chemical extraction. Thus, the complexation constant was determined, taking into account the variation of the physical extraction, from the results of Figure 6 . The corresponding Van't Hoff plot is given in Figure 8.

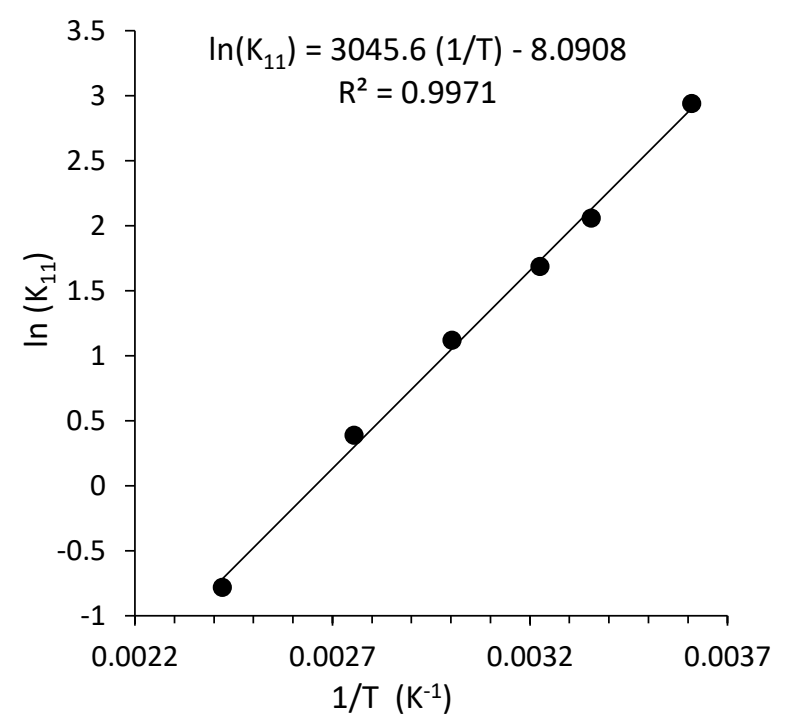

Figure 8: Van't Hoff plot of the complexation constant between 3-HP initially present in aqueous phase and TOA in organic phase

The global apparent enthalpy change of the complexation reaction is negative around $-25.3 \mathrm{~kJ}^{\mathrm{mol}}{ }^{-1}$ while the entropy change is $-67.3 \mathrm{~J} \cdot \mathrm{mol}^{-1} \cdot \mathrm{K}^{-1}$. Indeed, the ion pair formation through proton transfer and solvation is expected to be exothermic [23] due to stabilizing electrostatic interactions and specific solvation like H-bonding in solution. For example, Tamada and King [23] found that the complexation of lactic acid by Alamine 336 in chloroform, providing specific $\mathrm{H}$-bonding, was twice more exothermic than in methyl isobutyl ketone. The formation of a single complex out of 2 species as well as the 
formation of specific $\mathrm{H}$-bonds reduces the entropy of the system and this explains the negative entropy change of reaction. Contrary to the physical extraction, the reactive extraction is an enthalpy-driven process. This totally different thermodynamic behavior is in line with the 3-HP extraction mechanisms by physical and reactive extractions discussed in a previous work [8]. Given the converse temperature dependence of these two mechanisms, it is expected that they would annihilate each other at a given temperature. By extrapolating our experimental data on a larger temperature range, as shown in Figure 9, we found that the minimum extraction yield should be obtained at around $185^{\circ} \mathrm{C}$, supposing that dilute 3-HP solutions do not decompose under such conditions. Further increasing the temperature would be useless to reverse the extraction efficiency. At $140^{\circ} \mathrm{C}$, no degradation products like acrylic acid were found in the aqueous phase.

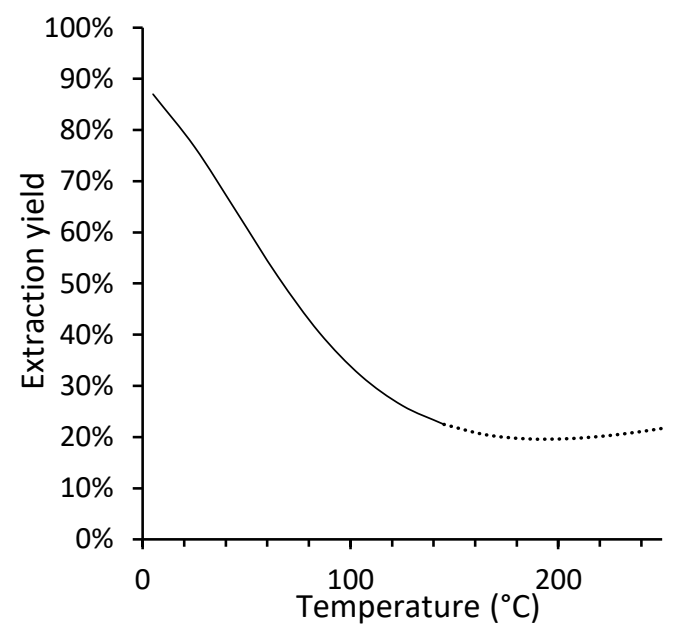

Figure 9: Extraction yield of a $5 \mathrm{~g} / \mathrm{L}$ 3-HP solution calculated from the thermochemical parameters (extrapolated above $140^{\circ} \mathrm{C}$ )

These results give valuable inputs for the design of temperature swing back-extraction process. For example, the reactive extraction can be performed at room temperature, then a cold washing of the organic phase can be performed to remove the species other than the acid (coextracted mainly by partition). Finally, a back-extraction at high temperature allows the recovery of the purified acid in an aqueous phase. A simplified process diagram is proposed in figure 10. 


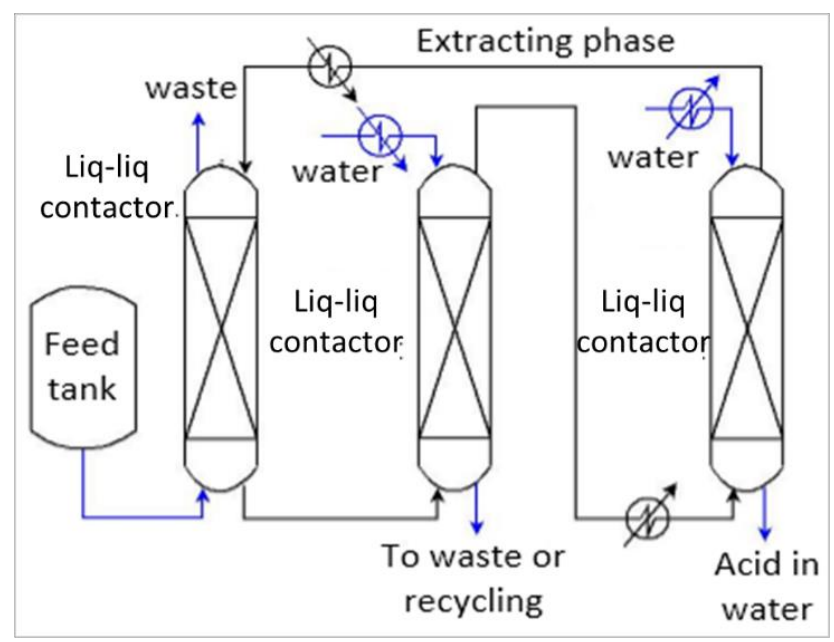

Figure 10: Simplified process diagram of extraction and back-extraction using temperature swing with a purification step with cold water in-between.

Finally, tuned combinations among the processes described herein are possible. For example, temperature and diluent swings can be coupled by heating the streams after the addition of the antisolvent [23], which should enhance the final 3-HP recovery yield. Another example is the combination of the temperature swing with the use of sodium hydroxide. This is the case of a process developed by Corbion Purac for the production of polymer grade lactic acid where the organic phase is backextracted using temperature swing (including the purification step) and then totally regenerated using sodium hydroxide (including a washing step) [35].

\section{Conclusion}

This study explored several strategies for the back-extraction of organic acids from loaded organic phases and reports pioneering experimental results for the effective recovery of 3-HP. Given the actual potential of 3-HP as a platform molecule for the production of commercially valuable chemicals, and the lack of data regarding its extraction and recovery, these results are valuable for designing its extraction and recovery process. 3-HP recovery from a loaded organic phase made of trioctylamine in decanol was studied. Using a salt without acid-base properties was shown to be feasible but a total recovery needs a high excess of salt and the trioctylamine, loaded with the anion of the salt, is not regenerated. Mineral bases such as sodium hydroxide or carbonate are thus advised because they are able to totally back-extract 3-HP in stoichiometric proportions while totally regenerating the amine 
extractant. However, the acid is recovered as its sodium salt, which may necessitate further processing steps to recover the acid under its non-dissociated form. Diluent swing with addition of hexane was proved to be effective in the case of 3-HP. Compared to other acids from the literature (like propionic acid) when the initial solvent is an alcohol, the diluent swing was unexpectedly efficient probably due to the higher hydrophilic character of 3-HP. The use of $60 \% \mathrm{v} / \mathrm{v}$ hexane with $1: 1$ volume ratio between the aqueous and the final organic phase led to 3 -HP recovery up to $70 \%$ but the solution was very dilute compared to the initial organic phase without hexane addition. Nonetheless, 3-HP was recovered in its non-dissociated form. Finally, temperature swing was performed over a large range of temperature $\left(4-140^{\circ} \mathrm{C}\right)$, and the higher the temperature the higher the 3-HP recovery. For example at $140{ }^{\circ} \mathrm{C}$, around $78 \%$ of the $3-\mathrm{HP}$ is found in the aqueous phase and no degradation products are found. Thermochemical calculations tend to show that further increasing the temperature would be useless to recover more 3-HP. Thermal regeneration is particularly interesting as the acid is not very diluted in the back-extraction stream, there is no chemical addition required and 3-HP is recovered under its non-dissociated form. All the methods addressed here and combinations thereof can be considered for the implementation of an efficient back-extraction process, performed experiments providing useful data for further process design and evaluation.

\section{Acknowledgement}

The authors would like to thank Cristian Felipe Puentes Mancipe for thermodynamic calculations concerning bubble points estimation and are grateful to Région Grand-Est, the Conseil Départemental de la Marne and Grand Reims for financial support. 


\section{References}

[1] T. Werpy, G. Petersen, A. Aden, J. Bozell, J. Holladay, J. White, A. Manheim, D. Elliot, L. Lasure, S. Jones, M. Gerber, K. Ibsen, L. Lumberg, S. Kelley, Top Value Added Chemicals from Biomass, Volume 1-Results of Screening for Potential Candidates from Sugars and Synthesis Gas, U.S. Department of Energy, Oak Ridge, TN, 2004.

[2] V. Kumar, S. Ashok, S. Park, Recent advances in biological production of 3-hydroxypropionic acid, Biotechnol Adv. 31 (2013). doi:10.1016/j.biotechadv.2013.02.008.

[3] A.S. Kertes, C.J. King, Extraction Chemistry of Fermentation Product Carboxylic Acids, Biotechnol. Bioeng. 28 (1986) 269-282.

[4] F. Chemarin, M. Moussa, M. Chadni, B. Pollet, P. Lieben, F. Allais, I.C. Trelea, V. Athès, New insights in reactive extraction mechanisms of organic acids: An experimental approach for 3hydroxypropionic acid extraction with tri-n-octylamine, Sep. Purif. Technol. 179 (2017) 523532. doi:10.1016/j.seppur.2017.02.018.

[5] M. Moussa, G. Burgé, F. Chemarin, R. Bounader, C. Saulou-Bérion, F. Allais, H.-E. Spinnler, V. Athès, Reactive extraction of 3-hydroxypropionic acid from model aqueous solutions and real bioconversion media. Comparison with its isomer 2-hydroxypropionic (lactic) acid, J. Chem. Technol. Biotechnol. 91 (2015) 2276-2285. doi:10.1002/jctb.4813.

[6] G. Burgé, F. Chemarin, M. Moussa, C. Saulou-Bérion, F. Allais, H.-É. Spinnler, V. Athès, Reactive extraction of bio-based 3-hydroxypropionic acid assisted by hollow-fiber membrane contactor using TOA and Aliquat 336 in $n$-decanol, J. Chem. Technol. Biotechnol. 91 (2016) 2705-2712. doi:10.1002/jctb.4878.

[7] M. Matsumoto, M. Shibazaki, K. Kondo, Salting-out Extraction of 3-Hydroxypropionic Acid with Reactive Extraction and Aqueous Two-phase Systems, Solvent Extr. Res. Dev. 24 (2017) 141147.

[8] F. Chemarin, M. Moussa, F. Allais, V. Athès, I.C. Trelea, Mechanistic modeling and equilibrium prediction of the reactive extraction of organic acids with amines: A comparative study of two complexation-solvation models using 3-hydroxypropionic acid, Sep. Purif. Technol. 189 (2017) 475-487. doi:10.1016/j.seppur.2017.07.083.

[9] M. Puttemans, L. Dryon, D.L. Massart, Extraction of organic acids by ion-pair formation with trin-octylamine: Part 2. Back-extraction, Anal. Chim. Acta. 161 (1984) 381-386.

[10] L.J. Poole, C.J. King, Novel Regenerated Solvent Extraction Processes for the Recovery of Carboxylic Acids or Ammonia from Aqueous Solutions Part I. Regeneration of Amine-Carboxylic Acid Extracts, Lawrence Berkeley Laboratory, CA (USA), United States, 1990.

[11] V.M. Yabannavar, D.I.C. Wang, Extractive fermentation for lactic acid production, Biotechnol. Bioeng. 37 (1991) 1095-1100.

[12] A. Keshav, K.L. Wasewar, Back extraction of propionic acid from loaded organic phase, Chem. Eng. Sci. 65 (2010) 2751-2757. doi:10.1016/j.ces.2010.01.010.

[13] L. Ahsan, M.S. Jahan, Y. Ni, Recovery of Acetic Acid from the Prehydrolysis Liquor of Kraft Based Dissolving Pulp Production Process: Sodium Hydroxide Back Extraction from the Trioctylamine/Octanol System, Ind. Eng. Chem. Res. 52 (2013) 9270-9275. doi:10.1021/ie401285v.

[14] A. Krzyzaniak, B. Schuur, A.B. de Haan, Equilibrium studies on lactic acid extraction with N,Ndidodecylpyridin-4-amine (DDAP) extractant, Chem. Eng. Sci. 109 (2014) 236-243. doi:10.1016/j.ces.2014.01.030.

[15] T. Brouwer, M. Blahusiak, K. Babic, B. Schuur, Reactive extraction and recovery of levulinic acid, formic acid and furfural from aqueous solutions containing sulphuric acid, Sep. Purif. Technol. 185 (2017) 186-195. doi:10.1016/j.seppur.2017.05.036.

[16] A. Krzyżaniak, M. Leeman, F. Vossebeld, T.J. Visser, B. Schuur, A.B. de Haan, Novel extractants for the recovery of fermentation derived lactic acid, Sep. Purif. Technol. 111 (2013) 82-89. doi:10.1016/j.seppur.2013.03.031. 
[17] Y. Tong, M. Hirata, H. Takanashi, T. Hano, Back extraction of lactic acid with microporous hollow fiber membrane, J. Membr. Sci. 157 (1999) 189-198.

[18] I.M. Coelhoso, P. Silvestre, R.M.C. Viegas, J. Crespo, M.J.T. Carrondo, Membrane-based solvent extraction and stripping of lactate in hollow-fibre contactors, J. Membr. Sci. 134 (1997) 19-32.

[19] N.L. Ricker, C.J. King, Solvent Extraction of Wastewaters from Acetic-Acid Manufacture, Robert S. Kerr Environmental Research Laboratory (U.S. Environmental Protection Agency), Ada, OK, United States, 1980.

[20] T. Harington, M.M. Hossain, Extraction of lactic acid into sunflower oil and its recovery into an aqueous solution, Desalination. 218 (2008) 287-296. doi:10.1016/j.desal.2007.02.024.

[21] J. Gorden, T. Zeiner, G. Sadowski, C. Brandenbusch, Recovery of cis,cis-muconic acid from organic phase after reactive extraction, Sep. Purif. Technol. 169 (2016) 1-8. doi:10.1016/j.seppur.2016.05.032.

[22] K.L. Wasewar, A.B.M. Heesink, G.F. Versteeg, V.G. Pangarkar, Intensification of conversion of glucose to lactic acid: equilibria and kinetics for back extraction of lactic acid using trimethylamine, Chem. Eng. Sci. 59 (2004) 2315-2320. doi:10.1016/j.ces.2003.11.023.

[23] J.A. Tamada, C.J. King, Extraction of Carboxylic Acids with Amine Extractants. 3. Effect of Temperature, Water Coextraction, and Process Considerations, Ind. Eng. Chem. Res. 29 (1990) 1333-1338.

[24] M. Matsumoto, T. Takahashi, K. Fukushima, Synergistic extraction of lactic acid with alkylamine and tri-n-butylphosphate: effects of amines, diluents and temperature, Sep. Purif. Technol. 33 (2003) 89-93. doi:10.1016/S1383-5866(03)00002-9.

[25] E.Z. Lee, Y. Suk Huh, Y.-S. Jun, H. Jin Won, Y.K. Hong, Effect of Operating Variables on Backextraction Characteristics of Succinic Acid from Organic Phase, Biotechnol. Bioprocess Eng. 13 (2008) 342-346.

[26] M.G. Sadaka, A.A. Garcia, The Effect of Temperature on Forward/Back Extraction Using an Amine Extractant for the Recovery of Cyclic Hydroxy Carboxylic Acids, Sep. Sci. Technol. 33 (1998) 1667-1680. doi:10.1080/01496399808545072.

[27] D. Pal, A. Tripathi, A. Shukla, K.R. Gupta, A. Keshav, Reactive Extraction of Pyruvic Acid Using Tri-n-octylamine Diluted in Decanol/Kerosene: Equilibrium and Effect of Temperature, J. Chem. Eng. Data. 60 (2015) 860-869. doi:10.1021/je500964e.

[28] D.H. Han, Y.K. Hong, W.H. Hong, Separation characteristics of lactic acid in reactive extraction and stripping, Korean J. Chem. Eng. 17 (2000) 528-533.

[29] D. Yankov, J. Molinier, J. Albet, G. Malmary, G. Kyuchoukov, Lactic acid extraction from aqueous solutions with tri-n-octylamine dissolved in decanol and dodecane, Biochem. Eng. J. 21 (2004) 63-71. doi:10.1016/j.bej.2004.03.006.

[30] H. Huang, S.-T. Yang, D.E. Ramey, A Hollow-Fiber Membrane Extraction Process for Recovery and Separation of Lactic Acid from Aqueous Solution, Appl. Biochem. Biotechnol. 114 (2004) 671-688. doi:10.1385/ABAB:114:1-3:671.

[31] Y. Marcus, The properties of solvents, Wiley, Chichester ; New York, 1998.

[32] B. Gestblom, J. Sjöblom, Dielectric Relaxation Studies of Aqueous Long-chain Alcohol Solutions, Acta Chem. Scand. A. 38 (1984) 47-56.

[33] J.K. Vij, W.G. Scaife, J.H. Calderwood, The pressure and temperature dependence of the static permittivity and density of heptanol isomers, J. Phys. Appl. Phys. 11 (1978) 545.

[34] B. Gestblom, A. El-Samahy, J. Sjöblom, Dielectric relaxation studies of monoalcohol solutions with 1, 2-diols, J. Solut. Chem. 14 (1985) 375-392.

[35] B.I. Veldhuis-Stribos, J. Van Breugel, W.J. Groot, B.M. Dierdorp, Continuous process for preparing lactic acid, WO 01/27064 A1, 2001. 